\title{
Special Issue on Fun with Algorithms
}

\author{
Paolo Boldi · Luisa Gargano
}

Published online: 21 September 2011

(C) Springer Science+Business Media, LLC 2011

...pleasure has probably been the main goal all along. But I hesitate to admit it, because computer scientists want to maintain their image as hard-working individuals who deserve high salaries. Sooner or later society will realise that certain kinds of hard work are in fact admirable even though they are more fun than just about anything else.

Donald E. Knuth

Whether we are ready to confess it or not, fun makes up a large part of the motivations behind our work as scientists. The fact that research can be fun is something unusual, even slightly difficult to understand for an outsider: science in general, and computer science in particular, gets definitely a bad press and has little charm. Computer science scholars are popularly identified with a bunch of bored, competitive nerds that work hard and rarely have fun. We all know this is far from true: most of us started to do research for fun, and fun is still often the spring of our talent. Although we like to keep this secret, sometimes applications come afterwards, whereas curiosity, amusement and enjoyment come first. In this issue, we allowed recreation to come into the limelight and phantasy to gain its long-due praise.

Entertainment has been declined in various forms, according to different tastes and backgrounds, but we believe that all readers will be able to seize and share part of the authors' pleasure. Of course, fun does not mean superficiality: all the contributions

P. Boldi $(\bowtie)$

Dipartimento di Scienze dell'Informazione, Università degli Studi di Milano, via Comelico 39/41, 20135 Milano, Italy

e-mail: paolo.boldi@gmail.com

L. Gargano

Dipartimento di Informatica ed Applicazioni "Renato M. Capocelli”, Università degli Studi di Salerno, via Ponte Don Melillo, 84084 Fisciano (SA), Italy 
are absolutely far from being trivial, and many of the results presented turn out to have interesting potential applications, that most readers will also appreciate.

In some cases, the very inspiration of the papers come from a game or a wellknown puzzle (like in Christmas Gift Exchange Games or in The Feline Josephus Problem, that considers a generalization of a problem already studied by Girolamo Cardano in the 16th century). In other cases, the connection between games and IT real-world problems plays a central role: in On Approximate Jumbled Pattern Matching in Strings, for example, scrabble-like games are related to a form of approximate pattern matching (which, by the way, has important applications to bioinformatics); Guessing Bank PINs by Winning a Mastermind Game establishes an interesting relation between a generalization of Mastermind and bank-PIN guessing.

We all know, games are fun, and when the going gets tough the tough get going: otherwise said, a provably difficult game is probably more fun. This is the theme of The Complexity of Flood Filling Games and Simple Wriggling is Hard unless You Are a Fat Hippo (the former about a well-known game called Flood-It, and the second considering a snake moving in a polygonal domain with holes). Both games have a nice visual nature, that is certainly an inspiration by itself, like in the paper In-place Algorithm for Erasing a Connected Component in a Binary Image.

But games are not the only way to get entertained. Sometimes, real-life situations have naturally inspired the authors of our contributions, like dealing with bullies (in Scheduling with Bully Selfish Jobs) or needing good strategies to maintain privacy (in Maintaining Privacy on a Line). Finally, sometimes fun is a simple matter of phantasy (like Searching for Black Holes in Subways and Two Skew-Binary Numeral Systems and One Application).

We would like to thank the authors that contributed to this issue, not only for their competence, but also for their wit and sense of humor. We also owe our gratitude to all the reviewers, who carefully worked on the papers and often provided insightful comments and remarks. Finally, we thank all the people at Springer for helping us in our work. Most probably all the authors had fun in writing their papers, and certainly we did as editors; we hope that this issue will be fun for you to read, too. 